\title{
Few-Body Problems in Hadron Spectroscopy *
}

\author{
Jean-Marc Richard ${ }^{\mathrm{a}}$ \\ anstitut des Sciences Nucléaires, Université Joseph Fourier-CNRS-IN2P3, \\ 53, avenue des Martyrs, 38026 Grenoble Cedex, France
}

\begin{abstract}
Some rigorous results can be derived using a very simple approach to hadron spectroscopy, in which a static potential is associated with non-relativistic kinematics. Several regularities of the experimental spectrum are explained by such models. It is underlined that certain methods developed for hadronic physics have found applications in other fields, in particular atomic physics. A few results can be extended to cases involving spin-dependent forces or relativistic kinematics.
\end{abstract}

\section{Introduction}

The dynamics of light quarks is presumably very intricate, with non-perturbative effects even at short distances, and highly-relativistic motion of the constituents inside hadrons. Nevertheless, it is interesting to consider a fictitious world, where the quark dynamics is governed by a simple Hamiltonian with non-relativistic kinematics supplemented by a static, flavour-independent potential. Most regularities derived from the properties of the Schrödinger equation are observed in the experimental spectrum. This suggests that the QCD theory of quark confinement should lead to similar regularities.

Several results derived in the context of quark models have been successfully applied to other few-body problems, for instance in atomic physics. Examples will be given in this review.

A challenge consists of extending the theorems on level order, convexity, etc., to less naive Hamiltonians with spin-dependent forces and relativistic kinematics. Some of the first results will be mentioned.

\section{Results on mesons}

Studies of the quark model have been stimulated by the discovery of $J / \Psi$ and $\Upsilon$ resonances and their excitations and, in particular, the simultaneous description of both spectra using the same potential (flavour independence). Many rigorous results have been summarized in the reviews by Quigg and Rosner [1] and by Martin and Grosse [2,3]. A few examples are given in this section, dealing with energy levels. A result on the wave function will be mentioned in Sec. 9 .

\footnotetext{
*Invited Talk at the 17th European Conference on Few-Body Physics, 11-16 September 2000, Evora,
} Portugal, to appear in Nuclear Physics A 
Current potentials reproduce the observed pattern that $E(1 P)<E(2 S)$. Within the notation $(n, \ell)$ adopted here for quarkonium, the radial wave function has $n-1$ nodes and the principal quantum number of atomic physics is $n+\ell$. It has been proved that $E(n+1, \ell)>E(n, \ell+1)$ if $\Delta V>0$, and the reverse if $\Delta V<0$. The Coulomb degeneracy is recovered as a limiting case. From the Gauss theorem, the sign of the Laplacian $\Delta V=r^{-2}\left[r^{2} V^{\prime}\right]^{\prime}$ reflects whether the charge $Q(r)$ seen at distance $r$ grows (asymptotic freedom), decreases or remains constant.

This "Coulomb theorem" can be applied successfully to muonic atoms, which are sensitive to the size of the nucleus $(Q(r) \nearrow)$, and to alkaline atoms whose last electron penetrates the inner electron shells $(Q(r) \searrow)$. The latter property also hold for the metastable $\bar{p}-$ He molecules described by Korobov at this conference [4].

Another theorem explains how the harmonic oscillator (h.o.) degeneracy $E(n+1, \ell)=$ $E(n, \ell+2)$ is broken. A strict inequality is obtained if the sign of $V^{\prime \prime}$ is constant [3].

In both the complete Hamiltonian $\mathbf{p}_{1}^{2} /\left(2 m_{1}\right)+\mathbf{p}_{2}^{2} /\left(2 m_{2}\right)+V\left(r_{12}\right)$ or its reduced version $\mathbf{p}^{2} /(2 \mu)+V(r)$, the individual inverse masses, $m_{i}^{-1}$, or the inverse reduced mass, $\mu^{-1}$, enter linearly through a positive operator $\mathbf{p}^{2}$. This implies that each energy level is an increasing function of this inverse mass, and that the ground-state energy (or the sum of first levels) is a concave function of this variable [5]. Let us mention two among the many applications.

The dynamics of heavy quarkonia $Q \bar{Q}$ is very sensitive to the change from $Q=c$ to $Q=b$, while open-flavour mesons are dominated by the light quark mass. Thus $B-D$ is essentially the difference between quark masses $b-c$, while the effect of this difference is reduced in $\Upsilon-\Psi$ by the change in the binding energy. After removing or estimating the hyperfine effects, one gets from the data [6]

$$
\frac{1}{2}(\Upsilon-\Psi) \simeq 3.2 \mathrm{GeV}<(B-D) \simeq 3.3 \mathrm{GeV}
$$

The second example is concerned with comparing symmetric mesons $Q \bar{Q}$ with hidden flavour to asymmetric mesons $Q^{\prime} \bar{Q}$ with open flavours. As $(b \bar{c})$, for instance, has an inverse reduced mass which is the average between that of $(b \bar{b})$ and that of $(c \bar{c})$, then [0],8] $(b \bar{c})>(b \bar{b}+c \bar{c}) / 2$ is expected. Moreover, $\mathrm{d} E / \mathrm{d} \mu^{-1}=\left\langle\mathbf{p}^{2}\right\rangle / 2$, and this latter matrix element can be estimated from the excitation spectrum and bounded along its path from $(b \bar{b})$ or $(c \bar{c})$ to $(b \bar{c})$. One eventually ends with a rigorous window for the mass of the spin-averaged ground state [3,9]

$$
6.26<(b \bar{c})<6.43 \mathrm{GeV} \text {. }
$$

Of course, hyperfine effects should be added in more precise tests. The accuracy of the first measurement [6]

$$
(b \bar{c})=6.4 \pm 0.4 \mathrm{GeV}
$$

is not yet sufficient to test flavour independence.

Convexity in the inverse reduced mass also implies that $\left(b \bar{q}_{2}\right)+\left(c \bar{q}_{1}\right)<\left(\bar{q}_{1}\right)+\left(c \bar{q}_{2}\right)$ if $b>c$ and $q_{2}>q_{1}$. In particular,

$$
(b \bar{c})>(b \bar{s})+(c \bar{c})-(c \bar{s}) \simeq 6.52 \mathrm{GeV},
$$


which is less efficient but much more easily obtained than the r.h.s. of Eq. (2).

Note that an inequality such as $\mathrm{D}_{2}+\mathrm{H}_{2} \leq 2 \mathrm{HD}$ should hold for variants of the hydrogen molecule involving isotopes, as they experience the same Born-Oppenheimer potential.

One of the "sides" of the Coulomb theorem can be adapted to the Klein-Gordon equation [3]. If $\Delta V \leq 0$, then $E(n+1, \ell)<E(n, \ell+1)$.

\section{Level order of baryon spectra}

For many years, the only widespread knowledge of the 3-body problem was the harmonic oscillator. This remains true outside the few-body community. The discussion on baryon excitations is thus often restricted to situations where $V=\sum v\left(r_{i j}\right)$, with $v(r)=K r^{2}+\delta v$, and $\delta v$ treated as a correction.

First-order perturbation theory is usually excellent, especially if the oscillator strength $K$ is variationally adjusted to minimise the magnitude of the corrections. However, when first-order perturbation is shown (or claimed) to produce a crossing of levels, one is reasonably worried about higher-order terms, and a more rigorous treatment of the energy spectrum becomes desirable.

In this context, it should be recalled that in the $N$ and $\Delta$ sector (not in the $\Lambda$ one!), the radial excitation with positive parity (Roper resonance) comes first, and the orbital excitation with positive parity just above. On the other hand, any simple potential such as $v(r)=\lambda r-a / r$ gives the opposite ordering. This problem has been known for many years. It has been vigorously revisited by Glozman and collaborators [10 and many others. The true nature of light-quark dynamics is outside the scope of this review, which will be focused on the level order in 3-body potential models.

A decomposition better than $V=\sum K r_{i j}^{2}+\delta v$ is provided by the generalised partialwave expansion

$$
V=V_{0}(\rho)+\delta V
$$

where $\rho \propto\left(r_{12}^{2}+r_{23}^{2}+r_{31}^{2}\right)^{1 / 2}$ is the hyperradius. The last term $\delta V$ gives a very small correction to the first levels [11]. With the hyperscalar potential $V_{0}$ only, the wave function reads $\Psi=\rho^{-5 / 2} u(\rho) P_{[L]}(\Omega)$, where the last factor contains the "grand-angular" part. The energy and the hyperradial part are governed by

$$
u^{\prime \prime}(\rho)-\frac{\ell(\ell+1)}{\rho^{2}} u(\rho)+m\left[E-V_{0}(\rho)\right] u(\rho)=0,
$$

very similar to the usual radial equations of the 2-body problem, except that the effective angular momentum is now $\ell=3 / 2$ for the ground-state and its radial excitations and $\ell=5 / 2$ for the first orbital excitation with negative parity. The Coulomb theorem holds for non-integer $\ell$. If $\Delta V>0$, then $E(2 S)>E(1 P)$, i.e., the Roper comes above the orbital excitation [12]. Note that a three-body potential cannot be distinguished from a simple pairwise interaction once it is reduced to its hyperscalar component $V_{0}$ by suitable angular integration. It also results from numerical tests that relativistic kinematics does not change significantly the relative magnitude of orbital vs. radial excitation energies.

The problem of the Roper resonance is thus a difficult one if one requires a model to reproduce perfectly the observed masses. Several solutions have been proposed, including 
accepting with philosophy that a $100 \mathrm{MeV}$ discrepancy on a particular level does not necessarily means the end of the conventional quark level |13. One might mention:

1. Introducing in the potential some terms with negative Laplacian. In particular, scalarmeson exchange induces a Yukawa-like potential $v \propto-\exp (-\mu r) / r$. Its strength is however not sufficient to change the level order, when it is associated with a linear potential of reasonable magnitude [14].

2. Introducing a specific spin or spin-flavour dependence in the potential, with the consequence that Eq. (6) is no longer valid: the effective potential in negative-parity states differs from the one governing the ground state and its radial excitations. This is what occurs with pion exchange in the models introduced by Glozman and others.

3. More drastic solutions consist of considering the Roper resonance as a multiquark state or a hybrid state, possibly mixed with conventional radial excitations. The production and decay properties do not provide much support for such an interpretation.

Back to spectral theory, the splitting of levels in the nearly hyperscalar potential (5) is very similar to the famous pattern of the $N=2$ h.o multiplet [15, 16], except that the Roper is disentangled [17,11]. A similar result is found for higher negative-parity excitation: the split $N=3$ levels of the nearly harmonic model are separated into a radially excited $L=1$ and a set of split $L=3$ levels. Details and references can be found in Refs. 18,17,11.

\section{Test of flavour independence for baryons}

A look at the baryon spectrum gives guide lines to our intuition which are contradictory. We have just seen that the "vertical spectrum", i.e., the ordering of radial vs. orbital excitations suggests flavour-dependent potentials such as those mediated by exchange of Goldstone bosons. On the other hand, the "horizontal spectrum", i.e., the spectrum of ground states with different flavour content, is rather smooth. There is a very continuous and slow increase in mass when a strange quark replaces an ordinary one. Strangeness excitation usually costs less than spin excitation.

This latter observation suggests describing all ground states with the same universal (flavour independent) potential and then applying moderate spin corrections "à la DGG" [19], where flavour dependence occurs only through the mass of the constituent in the Breit-Fermi term. This was basically the substance of the Isgur-Karl model [13] and other attempts with non-harmonic confinement [20].

This leads to considering the mathematical properties of the 3-body spectrum in a given potential as a function of the constituent masses. We have seen in Sec. 2 that for any 2-body potential $V$ which is flavour independent (just required to produce bound states), inequalities such as $(b \bar{b})+(c \bar{c}) \leq 2(b \bar{c})$ can be derived. The analogue for baryons,

$$
(Q Q q)+\left(Q^{\prime} Q^{\prime} q\right) \leq 2\left(Q Q^{\prime} q\right)
$$

requires, however, mild restrictions, which are always satisfied by the potentials used in quark model, whatever quark masses are used for $Q, Q^{\prime}$ and $q$. Flavour independence is the only serious hypothesis. See Ref. [21].

For instance, the equal spacing rule, $\Omega^{-}-\Xi^{*}=\Xi^{*}-\Sigma^{*}=\Sigma^{*}-\Delta$, is understood as follows: the central force gives a concave behaviour, with for instance $\Omega^{-}-\Xi^{*}<\Xi^{*}-\Sigma^{*}$, 
but a quasi perfect linearity is restored by the spin-spin interaction which acts more strongly on light quarks. A similar scenario holds for the Gell-Mann-Okubo formula [20].

Inequalities can also be written for baryons with heavy flavour, some of them being more accessible than others to experimental checks in the near future. Examples are

$$
\begin{aligned}
& 3(b c s) \geq(b b b)+(c c c)+(s s s) \\
& 2(b c q) \geq(b b q)+(c c q), \quad 2(c q q) \geq(c c q)+(q q q)
\end{aligned}
$$

\section{Baryons with two heavy flavours}

There is a renewed interest in this subject [22,23]. The recent observation of the $(b \bar{c})$ mesons demonstrates our ability to reconstruct hadrons with two heavy quarks from their decay products.

Baryons with two heavy quarks $\left(Q Q^{\prime} q\right)$ are rather fascinating: they combine the adiabatic motion of two heavy quarks as in $J / \Psi$ and $\Upsilon$ mesons with the highly relativistic motion of a light quark as in flavoured mesons $D$ or $B$.

The wave function of $(Q Q q)$ exhibits a clear diquark clustering with $r(Q Q) \ll r(Q q)$ for the average distances. This does not necessarily mean that for a given potential model, a naive two-step calculation is justified. Here I mean: estimate first the $(Q Q)$ mass using the direct potential $v(Q Q)$ only, and then solve the $[(Q Q)-q]$ 2-body problem using a point-like diquark. If $v$ is harmonic, one would miss a factor $3 / 2$ in the effective spring constant of the $(Q Q)$ system, and thus a factor $(3 / 2)^{1 / 2}$ in its excitation energy.

On the other hand, it has been checked that the Born-Oppenheimer approximation works extremely well for these $(Q Q q)$ systems, even when the quark mass ratio $Q / q$ is not very large. This system is the analogue of $\mathrm{H}_{2}^{+}$in atomic physics.

\section{6. $S_{N}$-expansion and stability of multiquarks}

The deuteron and other nuclei are bound by long-range nuclear forces, not by direct quark-quark interaction, and thus cannot be considered as genuine multiquark states. Stable multiquarks have been searched for in all possible channels, with the exception of very exotic hadronic configurations with several heavy flavours, and the results are negative.

The absence, or at least the non-proliferation of stable multiquarks, is reproduced in most model calculations, even the simplest ones.

I shall not discuss too much here the multiquark states such as the hexaquark $H$ [24] or the pentaquark $P$ [25] whose binding is tentatively achieved by chromomagnetic forces. For these states, the predictions vary from one author to another, and the questions are: i) Is the one-gluon-exchange appropriate for describing hyperfine effects ? Note that the Goldstone-boson-exchange model gives slightly different predictions on multiquarks [26], as compared to the chromomagnetic model, but the main message remains identical: there is no proliferation of multiquarks in the spectrum.

ii) What is the value of the short-range correlation in a multiquark, as compared to ordinary hadrons? This quantity governs, indeed, the strength of chromomagnetic effects.

Another issue deals with the absence of collective binding due to chromoelectric (con- 
fining) forces. Consider a four-body problem with Hamiltonian

$$
\begin{aligned}
H_{4}(x) & =\sum_{i=1}^{4} \frac{\mathbf{p}_{i}^{2}}{2 m}+(1-2 x)\left(V_{12}+V_{34}\right)+(1+x)\left(V_{13}+V_{14}+V_{23}+V_{24}\right) \\
& =H_{\mathrm{S}}+x H_{\mathrm{MS}}
\end{aligned}
$$

where the parameter $x$ measures the departure from a fully symmetric interaction. From the variational principle, the ground-state energy $E(x)$ is maximal at $x=0$. Indeed, with obvious notation,

$$
E(x)=\langle\Psi(x)|H(x)| \Psi(x)\rangle \leq\langle\Psi(0)|H(x)| \Psi(0)\rangle=E(0),
$$

where the last step results from $\Psi(0)$ being symmetric and thus insensitive to the mixedsymmetry part, $H_{\mathrm{MS}}$. As $x$ enters $H$ linearly, $E(x)$ is a concave function [5] and thus should exhibit near $x=0$ a parabola-like shape. This means that

$$
E(0)>E\left(x_{1}\right)>E\left(x_{2}\right) \quad \text { if } \quad 0<x_{1}<x_{2}
$$

is guaranteed, and

$$
E\left(x_{1}^{\prime}\right)>E\left(x_{2}\right) \quad \text { if } \quad x_{1}^{\prime}<0<x_{2} \quad \text { and } \quad\left|x_{1}^{\prime}\right|<x_{2}
$$

is very likely. In short, a larger asymmetry leads to a lower algebraic energy.

Consider now the most primitive ansatz for the multiquark interaction, the so-called additive colour model

$$
V=-\frac{3}{16} \sum_{i<j} \tilde{\lambda}_{i}^{\mathrm{c}} \cdot \tilde{\lambda}_{j}^{\mathrm{c}} v\left(r_{i j}\right)=\sum_{i<j} g_{i j} v\left(r_{i j}\right) .
$$

This is a pairwise interaction with a colour dependence that corresponds to pure octet exchange. The normalisation is such that $v$ is the central potential for quarkonia. If this model is applied to four-quark configurations $(\bar{q} \bar{q} q q)$ assuming a frozen colour wave function, then when one looks at the distribution of the strength factors $g_{i j}$, one observes that the largest asymmetry is obtained for the meson-meson threshold, for which an appropriate labelling gives

$$
g_{i j}=0, \quad \text { except } \quad g_{12}=g_{34}=1 .
$$

It is easily checked that the strength distributions corresponding to a "true baryonium", with $(q q)$ in a $\overline{3}$ colour state, and $(\bar{q} \bar{q})$ in 3 colour state, or a "mock baryonium" state, with a $6-\overline{6}$ colour structure, are more clustered around the symmetry point where all $g_{i j}$ are equal to $1 / 3$. (Colour neutrality ensures that $\sum g_{i j}$ is the same for all colour-singlet configurations.)

Indeed, solving the equal-mass $(\bar{q} \bar{q} q q)$ problem using a frozen colour wave function and the potential (13) never leads to stable multiquarks.

Binding requires a combination of

- mixing of several colour wave functions

- spin-dependent effects

- a better estimate of the multiquark potential. Note however that the recent lattice 
estimate by Green et al. [27] suggests that the true 4-quark potential is less attractive than the ansatz (13).

- another type of $S_{N}$ breaking which would be favourable to multiquark binding.

This latter mechanism was suggested several years ago. Its most remarkable feature is that theorists agree on the main result, namely that $(\bar{q} \bar{q} Q)$ becomes stable in the limit where the quark mass ratio $Q / q$ becomes very large.

The Hamiltonian

$$
H=\frac{1}{2 m}\left(\mathbf{p}_{1}^{2}+\mathbf{p}_{2}^{2}\right)+\frac{1}{2 M}\left(\mathbf{p}_{3}^{2}+\mathbf{p}_{4}^{2}\right)+V
$$

with $V$ even under charge conjugation, i.e., simultaneous $(1 \leftrightarrow 3)$ and $(2 \leftrightarrow 4)$ exchanges, can be seen as the $x=1$ value of

$$
\begin{aligned}
& H(x)=H_{\text {even }}+x H_{\text {odd }}, \\
& H_{\text {even }}=\left(\frac{1}{4 m}+\frac{1}{4 M}\right) \sum_{i} \mathbf{p}_{i}^{2}+V, \\
& H_{\text {odd }}=\left(\frac{1}{4 m}-\frac{1}{4 M}\right)\left(\mathbf{p}_{1}^{2}+\mathbf{p}_{2}^{2}-\mathbf{p}_{3}^{2}-\mathbf{p}_{4}^{2}\right) .
\end{aligned}
$$

When $x$ grows from 0 to 1 , the meson-meson threshold is unchanged, because the inverse reduced mass of $(m, M)$ is already an average of $m^{-1}$ and $M^{-1}$. On the other hand, the four-quark energy $E(x)$ decreases. Stability of $(\bar{q} \bar{q} Q Q)$ becomes possible if $Q \gg q$.

Remarks on this multiquark are in order:

1. If the mass ratio approaches the critical value for stability, the wave function has large meson-meson components. For larger $Q / q$, the binding is more pronounced, and the system acquires another structure in space and colour. The two heavy quarks form a colour $\overline{3}$ source (as in every $\Xi_{Q Q}$ baryon), which in turn, form a colour singlet with the two antiquarks, as in every flavoured antibaryon $(\bar{Q} q q)$. One thus uses well established colour coupling. This contrasts with the highly speculative colour structure in "colourchemistry".

2. The same mechanism holds for $(+,+,-,-)$ configurations in atomic physics. The positronium molecule $\mathrm{P}_{\mathrm{S}_{2}}$ is very weakly bound. The hydrogen molecule, where different masses experience the same potential as $\mathrm{Ps}_{2}$, is much more stable. In fact the stability of $\mathrm{Ps}_{2}$, as first demonstrated in 1947 by Hylleraas and Ore [28], implies stability for hydrogen and any similar configurations such as $K^{+} K^{+} \pi^{-} \pi^{-}$[29]. On the other hand, the molecule $p \bar{p} e^{+} e^{-}$is unstable, even in the limit where strong interaction and electromagnetic annihilation is switched off: one can perform an $S_{N}$ expansion analogous to (16); the 4-body energy decreases as asymmetry $\left(m^{-1} \neq M^{-1}\right)$ is implemented, but the threshold benefits much more from this effect. In the limit where $M \gg m$, the system breaks in a point-like protonium which cannot attract the remaining positronium. The critical mass ratio for stability has been estimated to about $M / m \simeq 2.2$ [30.

\section{Hall-Post inequalities for mesons and baryons}

The simple additive model (13) gives for baryons the " $1 / 2$ " rule

$$
V_{q q q}=\sum_{i<j} V_{q q}\left(r_{i j}\right), \quad \text { with } \quad V_{q q}=\frac{1}{2} V_{q \bar{q}}
$$


or, for Hamiltonians, after distributing the kinetic energy among different terms

$$
H_{q q q}=\frac{1}{2} \sum_{i<j} H_{q \bar{q}}=\frac{1}{2} \sum_{i<j}\left[\frac{\mathbf{p}_{i}^{2}}{2 m}+\frac{\mathbf{p}_{j}^{2}}{2 m}+V_{q \bar{q}}\left(r_{i j}\right)\right] .
$$

The variational principle, using the baryon wave function gives

$$
E(q q q) \geq 3 E(q \bar{q}) / 2
$$

or for the masses, including the contribution of the constituents,

$$
(q q q) / 3 \geq(q \bar{q}) / 2
$$

In other words, matter is heavier per quark in baryons than in mesons. This is seen, e.g., by comparing $\Omega^{-}(1672)$ and $\phi(1020)$, where we are dealing with the spin-triplet potential, including the spin-spin term. Otherwise, one can compare the spin $1 / 2$ baryon to a suitable combination of spin 0 and spin 1 mesons.

Some generalisations to unequal quark masses are straightforward, for instance

$$
2(b c s) \geq(b \bar{c})+(c \bar{s})+(s \bar{b})
$$

On the other hand, if one rewrites (20) as

$$
(\bar{q} \bar{q} \bar{q})+(q q q) \geq 3(\bar{q} q)
$$

which expresses the fact that quark rearrangement is energetically allowed for annihilation at rest, one observes that for a large enough mass ratio $Q / q$, the inequality becomes inverted, namely

$$
(\overline{Q Q Q})+(q q q)<3(\bar{Q} q)
$$

For instance, a triple-charm antibaryon would not "annihilate" on ordinary matter.

These inequalities were rediscovered in quark model studies [31 33] but they were known from previous works on the stability of matter and the thermodynamic limit of $N$-body systems [34], and studies of few-nucleon systems [35].

The simplest result is the following. If

$$
H_{N}(m, g)=\sum_{i}^{N} \frac{\mathbf{p}_{i}^{2}}{2 m}+g \sum_{i<j} V\left(r_{i j}\right)
$$

governs a system of $N$ bosons and $E_{N}$ denotes the ground-state energy, then the identity

$$
H_{N}(m, g)=\frac{1}{N-2} \sum_{i} H_{N-1}^{(i)}\left(\frac{N-1}{N-2} m, g\right),
$$

leads to

$$
E_{N}(m, g) \geq \frac{N}{N-2} E_{N-1}\left(\frac{N-1}{N-2} m, g\right)
$$


This inequality is never saturated, because the overall translation energy of the $(N-1)$ body subsystems within the $N$-body system is neglected. An improvement consists of replacing the decomposition (25) by the identity [35,36]

$$
\widetilde{H}_{N}(m, g)=\frac{1}{N-2} \sum_{i} \widetilde{H}_{N-1}^{(i)}\left(\frac{N}{N-1} m, g\right),
$$

relating the translation-invariant Hamiltonians

$$
\widetilde{H}_{N}=H_{N}-\frac{\left[\sum \mathbf{p}_{i}\right]^{2}}{2 N m} .
$$

This leads to the new inequality

$$
E_{N}(m, g) \geq \frac{N}{N-2} E_{N-1}\left(\frac{N}{N-1} m, g\right) .
$$

As $N m /(N-1)$ is smaller than $(N-1) m /(N-2)$ for $N \geq 3$, the bound (29) is better than (26), since any binding energy in a given potential is a decreasing function of the constituent mass.

By recursion, an inequality such as (26) or (29) provides a bound on $E_{N}$ in terms of the 2-body energy $E_{2}$. In the case of the harmonic oscillator for bosons, the bound derived from (29) is saturated, whereas the bound derived from (26) is, by a factor $\sqrt{2}$, smaller than the exact result at large $N$.

The generalisation to different constituent masses has been carried out [37]. The decomposition of the Hamiltonian involves some free parameters which can be adjusted to optimise the inequality. Again, saturation is obtained in the case of harmonic forces.

The situation is far more difficult for fermions. In Ref. [34], the decomposition

$$
H_{N}(m, g)=\frac{1}{2} \sum_{i} \sum_{j \neq i}\left[\frac{\mathbf{p}_{j}^{2}}{(N-1) m}+g V\left(r_{j i}\right)\right]
$$

is written down, which expresses $H_{N}$ in terms of Hamiltonians with $(N-1)$ independent particles. For fermions, one obtains

$$
E_{N}(m, g) \geq \frac{N}{2} f_{N-1}(m(N-1), g),
$$

where $f_{N}$ is the cumulated energy of a system of $N$ independent fermions, a notation borrowed from Ref. [38]. In Eq. (31), the translation energy of the $(N-1)$-body subsystem is neglected.

Basdevant and Martin [38] succeeded in improving this inequality in the case of powerlaw potentials $V \propto r^{n}$ with $n>1$ or combinations of those with positive weights. They obtained saturation for any number $N$ of fermions in the harmonic limit $n=2$. Recently Juillet et al. [39] used group-theoretical considerations to analyse the structure of $N$-body wave function in terms of the permutation of a $(N-1)$-subset and derived the following inequality

$$
\begin{aligned}
E_{N}^{S}(m, g) & \geq \frac{N-1}{N(N-2)(2 S+1)} \times \\
& {\left[S(N+2 S+2) E_{N-1}^{S-1 / 2}\left(m, \frac{N g}{N-1}\right)+(S+1)(N-2 S) E_{N-1}^{S+1 / 2}\left(m, \frac{N g}{N-1}\right)\right] . }
\end{aligned}
$$

where $S$ is the spin of the $N$-fermion system. 


\section{Convergence of variational calculations}

When computing the binding energy of a baryon or a more complicated system in a given potential model, one is faced with the choice of a method for solving the few-body problem.

Almost all possible methods have been applied to baryons. We already mentioned the approximation of a perturbed oscillator. The hyperspherical expansion and the Faddeev equations have also been applied very convincingly. The approximation of Feshbach and Rubinow [40], which looks at the best function of the perimeter $\sum r_{i j}$, is not very accurate for baryons with linear confinement, while it turns out to be very powerful for weaklybound states with short-range interaction.

Many variational methods are base on a decomposition

$$
\Psi^{M}=\sum_{i=1}^{M} c_{i} \varphi_{i}(\alpha, \beta, \ldots),
$$

where the linear parameters $c_{i}$ are determined from a eigenvalue equation and the range parameters $\alpha, \beta, \ldots$ adjusted by suitable minimisation of the energy. For most choices of the basis functions $\varphi_{i}$, one achieves a decent convergence of the variational energy $E^{N}$ toward the exact energy $E$, with the consequence that the approximate variational wave function $\Psi^{N}$ overlaps better and better the exact one, i.e., [5]

$$
\left|\left\langle\Psi^{M} \mid \Psi\right\rangle\right| \rightarrow 1 \quad \text { as } \quad E^{M} \rightarrow E .
$$

However, the variational wave function $\Psi^{M}$ might differ appreciably from the exact $\Psi$ in regions of negligible contribution to the normalisation integral but of interest for physics. In particular, the value of the wave function for $r_{i j} \rightarrow 0$ governs the production rate, the electromagnetic and annihilation width of quarkonia, the contribution of $W$-exchange to the weak decay of flavoured mesons and baryons, the parity-violating effects in atoms, etc. A comparison of the estimates of the correlation coefficient

$$
\delta_{12}=\left\langle\Psi \mid \delta^{(3)}\left(\mathbf{r}_{12}\right) \Psi\right\rangle
$$

using different 3-body methods is rather interesting. It shows that:

1. The quality of the Faddeev wave function is impressive, even with a small number of partial waves included.

2. The hyperspherical formalism provides a slow but safe convergence for this quantity.

3. Some variational expansions of type (33) fail in reproducing $\delta_{12}$ ! This is observed in particular when the basis is built out of the successive eigenstates of the harmonic oscillator. (These wave functions fall off too rapidly at large separation. To keep the normalisation and the average energy correct, a distortion is introduced at small separation). 4. However, the expansion on a basis of Gaussians does not suffer from the same difficulties, as contributions of longer and longer range can always be introduced in the expansion.

\section{Bounds on short-range correlations in baryons}

As seen in the last section, it is rather difficult to calculate the 3-body (or higher) wave function with good zero-range properties. 
To avoid lengthy computations, one can estimate $\delta_{12}$ using a trick proposed by Schwinger for the 2-body problem [1] and generalised to larger systems [41,12]. The basic idea is that $A$ and $A_{B}=A+[H, B]$ have same expectation value within any eigenstate of $H$. A suitable choice of $B$ might make $\left\langle\Psi^{M}\left|A_{B}\right| \Psi^{M}\right\rangle$ converge faster than $\left\langle\Psi^{M}|A| \Psi^{M}\right\rangle$ to the exact $\langle\Psi|A| \Psi\rangle$ as the size $M$ of the basis is increased.

For a 2-body problem with a central potential $V(r)$, the operator $A=\delta^{(3)}(\mathbf{r})$ is replaced by $A_{B} \propto \mathrm{d} V / \mathrm{d} r$, which is much less sensitive to a local flaw of the wave function. In particular $\delta_{12}$ comes out exact for a linear potential, however bad is the trial wave function, provided it is normalised. One also understands why in such a purely linear potential, $\delta_{12}^{n}$ is the same for all $S$-states, independent of the radial number $n$. A better result has been established [3]: $\delta_{12}^{n}$ increases with $n$ if $V^{\prime \prime}>0$, and decreases if $V^{\prime \prime}<0$. For instance, current potentials $-a / r+b r$ explain why the leptonic coupling is smaller for radially excited quarkonia than for the ground state.

For baryons, $A_{B}$ contains also a small centrifugal term, besides the derivative of the full potential $V$ with respect to $r_{12}$. Cohen and Lipkin [43] have compared the $\Sigma$ and $\Xi$ hyperfine splittings in models where this is induced by a contact term. In other words, they compared $\delta_{12}$ in $(q q s)$ and $(s s q)$. On can also [44] derive bounds on $\delta_{12}$.

The short-range correlation between electrons in ordinary atoms, the annihilation probability of exotic molecules with positrons can also be calculated using the Schwinger rule.

\section{Acknowledgments}

I would like to thank very much the organizers for the stimulating atmosphere of this Conference, and John Cole for constructive comments.

\section{REFERENCES}

1. C. Quigg and J.L. Rosner, Phys. Rep. 56 (1979) 167.

2. H. Grosse, A. Martin, Phys. Rep. 60 (1979) 341.

3. H. Grosse and A. Martin, Particle physics and the Schrodinger equation, Cambridge, UK: Univ. Pr. (1997) 167 p. (Cambridge monographs on particle physics, nuclear physics and cosmology. 6).

4. V.I. Korobov, Contribution to this Conference.

5. See, e.g., W. Thirring, A Course in Mathematical Physics, Vol. 3: Quantum Mechanics of Atoms and Molecules (Springer Verlag, New York, 1981).

6. Particle Data Group, Review of Particle Properties, Phys. Rev. D45 (1992) 1; D46 (1992) $5210(\mathrm{E})$.

7. R.A. Bertlmann and A. Martin, Nucl. Phys. B168 (1980) 111.

8. S. Nussinov, Phys. Rev. Lett. 52 (1984) 966.

9. E. Bagan, H.G. Dosch, P. Gosdzinsky, S. Narison and J.-M. Richard, Z. Phys. C64 (1994) 57.

10. L. Y. Glozman, Nucl. Phys. A663 (2000) 103.

11. J.-M. Richard, Phys. Rep. 212 (1992) 1.

12. H. Høgaasen and J.-M. Richard, Phys. Lett. B124 (1983) 520.

13. N. Isgur, "Critique of a pion exchange model for interquark forces," nucl-th/9908028.

14. P. Stassart, F. Stancu and J. M. Richard, J. Phys. G G26 (2000) 397. 
15. D. Gromes and I.O. Stamatescu, Z. Physik C3 (1979) 43.

16. N. Isgur and G. Karl, Phys. Lett. 72B (1977) 109; 74B (1978) 353;

Phys. Rev. D18 (1978) 4187.

17. J.-M. Richard and P. Taxil, Nucl. Phys. B329 (1990) 310.

18. F. Stancu and P. Stassart, Phys. Lett. B269 (1991) 243.

19. A. de Rújula, H. Georgi and S.L. Glashow, Phys. Rev. D12 (1975) 2060.

20. J.-M. Richard and P. Taxil, Ann. Phys. (N.Y.) 150 (1983) 267.

21. S. Nussinov, Phys. Rev. Lett. 52 (1984) 966;

J.-M. Richard and P. Taxil, Phys. Rev. Lett. 54 (1985) 847;

E. Lieb, Phys. Rev. Lett. 54 (1985) 1987;

A. Martin, J.-M. Richard and P. Taxil, Phys. Lett. 176B (1986) 224.

22. M.J. Savage and M.B. Wise, Phys. Lett. B248 (1990) 177; M.J. Savage and R. P. Springer, Int. J. Modern Phys. A6 (1991) 1701.

23. S. S. Gershtein, V. V. Kiselev, A. K. Likhoded and A. I. Onishchenko, Phys. Rev. D62 (2000) 054021.

24. R.L. Jaffe, Phys. Rev. Lett. 38, 195 (1977).

25. H.J. Lipkin, Phys. Lett. 195B, 484 (1987);

C. Gignoux, B. Silvestre-Brac and J.-M. Richard, Phys. Lett. 193B, 323 (1987).

26. F. Stancu, "Stability of multiquark systems," International Workshop on Hadron Physics: Effective Theories of Low Energy QCD, Coimbra, Portugal, 10-15 Sep 1999. hep-ph/9910547.

27. A. M. Green, J. Koponen and P. Pennanen, Phys. Rev. D61 (2000) 014014.

28. E.A. Hylleraas and A. Ore, Phys. Rev. 71 (1947) 493.

29. J.-M. Richard, J. Fröhlich, G.M. Graf, and M. Seifert, Phys. Rev. Lett. 71 (1993) 1332.

30. D. Bressanini, M. Mella and G. Morosi, Phys. Rev. A 55 (1997) 200.

31. S. Nussinov, Phys. Rev. Lett. 51 (1983) 2081.

32. J.-P. Ader, J.-M. Richard and P. Taxil, Phys. Rev. D25 (1982) 2370.

33. J.-M. Richard, Phys. Lett. B139 (1984) 408.

34. M.E. Fischer and D. Ruelle, J. Math. Phys. 7 (1966) 260; F.J. Dyson and A. Lenard, ibidem 8 (1967) 423; J.M. Lévy-Leblond, ibidem 10 (1969) 806.

35. R.L. Hall and H.R. Post, Proc. Phys. Soc. 90 (1967) 381.

36. J.-L. Basdevant, A. Martin and J.-M. Richard, Nucl. Phys. B343 (1990) 60.

37. J.-L. Basdevant, A. Martin, J.-M. Richard and T.T. Wu, Nucl. Phys. B 393 (1993) 111; A. Benslama, A. Metala, A. Bachkhaznadji, S.R. Zouzou, A. Krikeb, J.-L. Basdevant, J.-M. Richard, and T.T. Wu, Few-Body Systems 24 (1998) 24.

38. J.-L. Basdevant and A. Martin, J. Math. Phys. 37 (1996) 5916.

39. O. Juillet, S. Fleck, L. Theussl, J. Richard and K. Varga, "New Lower Bound on Fermion Binding Energies," quant-ph/0008048.

40. H. Feshbach and S.I. Rubinow, Phys. Rev. 98 (1955) 188.

41. J. Hiller, J. Sucher and G. Feinberg, Phys. Rev. A18 (1978) 2399.

42. M. Hoffmann-Ostenhof, T. Hoffman-Ostenhof and W. Thirring, J. Phys. B11 (1978) L571.

43. I. Cohen and H.J. Lipkin, Phys. Lett. B106 (1981) 119.

44. J.-M. Richard, Phys. Lett. B255 (1991) 435. 